Accelerator Department:

BROOKHAVEN NATIONAI LABORATORY

Associated Universities, Inc.

Upton, L.I., N.Y.

AGS DIVISION TECHNICAL NOTE

No. 31

R.R...Adams"s

February $15 ; 1967$

AGS OPERATIONS :SUMMARY FOR 1966

The AGS performance continues to improve. During 1966 , the AGS was on $4,991.27$ hours for experimenters and 680.62 hours for accelerator research. This tota 1 on time of $5,671.89$ hours is $75.8 \%$ of the scheduled time $(7,484.00$ hours $)$ for the year.

The tota 1 machine downtime of $24.2 \%$ is an improvement over the 1965 performance (28.5\%).. The AGS machine failures reduced from $12.1 \%$ for 1965 to $11.1 \%$ for 1966 and scheduled maintenance was reduced from $9.9 \%$ in 1965 to $7.2 \%$ in 1966

The total integrated machine use time for 1966 amounts to 19,000 hours, as compared to 12,000 hours for 1965 and 6000 hours for 1964 . More experiments were run in parallel than in the previous year.. During this year the 30-in. bubble chamber took $3,170,163$ pictures and the 80 -in. bubble chamber took $1,517,772$ pictures.

One 30 day shutdown was used to change experimenta 1 setups and to improve and maintain the machine. A short shutdown (5 days) was used to change one... experiment and:a third shutdown (6 days) was used to repair a faulted machine magnet. 
AGS OPERATIONS SUMMARY - CALENDAR YEAR $1966 \ldots \ldots \ldots \ldots \ldots \ldots \ldots \ldots \ldots$

EXPERTMENTAL OPERATIONS SUMMARY - CALENDAR YEAR 1966.......... 4 AGS OPERATIONS SUMMARY - MONTHLY $1966 \ldots \ldots \ldots \ldots \ldots \ldots \ldots \ldots \ldots$ AGS OPERATIONS SUMMARY - WEEKLY $1966 \ldots \ldots \ldots \ldots \ldots \ldots \ldots \ldots \ldots \ldots$ AGS ON TTME (hours) for HIGH ENERGY PHYSICS and MACHINE STUDIES ...1I - 12 AGS ON TIME $(\%)$ for HIGH ENERGY PHYSICS and MACHINE STUDIES ....13 - 14

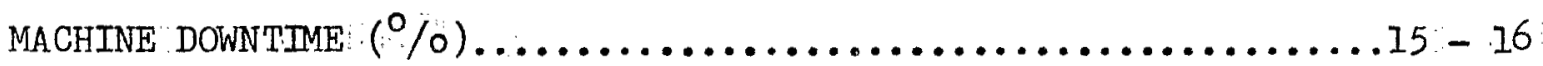

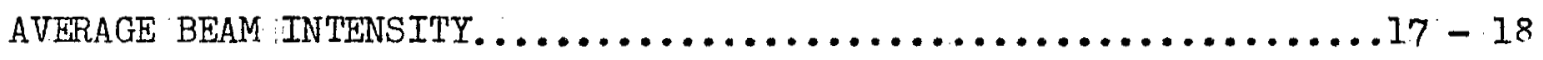
EXPERTMENTAL USERS SUMMARY - 30 inch BUBBLE CHAMBER ............ 19 - 20 EXPER IMENTAL USERS SUMMARY - 80 inch BUBBLE CHAMBER ..........2I - 22 EXPERTMENTAL USERS SUMMARY - COUNTER EXPERTMENTS. . . . . . . . . 23 - 24 AGS OPERATIONS SUMMARY - 1964, 1965, 1966 YEARIY COMPARISONS ..... 25 AGS. OPERATIONS SUMMARY - 1964, 1965, 1966 1/2 YEAR COMPARISONS.... 26 


\section{AGS ON}

1. For Experimenters

2. For Accelerator Research \%..

\section{Machine Downtime}

1. Start-up, Set-up, Shutdown

2. Requested by Experimenters

3. Experimental Set-up

4. Machine Failures

5. EAO Equipment Failures

6. Scheduled Maintenance.

Scheduled Operations:

Holidays

Shutdowns $\frac{\text { Hours }}{5,671.89} \quad \frac{\%}{75.8}$

$4,991.27 \quad 66.7$

$680.62 \quad 9.1$

$1,812: 11: \quad 24: 2$

$273.04 \quad 3.7$

$58.17 \quad 0.8$

31.75: $\quad 0.4$

$833.22 \quad 11.1$

$76.02 \quad 1.0$

539.91 $\quad 7.2$

$7,484.00 \quad 100.0$

$$
212.00
$$

$1,064: 00$

\section{AGS Performance}
a. Magnet pulses
$8,916,874$
b. Accelerated pulses
$7,481,254$
c. Missed pulses
d. Accelerated protons ( $p$ )
538,542
e. Average protons per Accelerated puise $(\alpha / b)$
$8.7 \times 10^{18}$
$11.6 \times 10^{11}$
f. Overall average protons per pulse $(d / b+c)$ 
Total AGS On Time

$5,671.89$ hours

1. For Experimenters

$4,991.27$ hours

2. For Accelerator Research 680.62 , hours :

Integrated Machine Use Time

19.008.88 hours

1. Integrated Experimental time

$18,082,38$ hours

2. Beam tests, etc. 229.95 hours

3. Accelerator Research 680.62 hours

4. Radiation Measurements

15.93 hours

Total $80^{\circ}$ inch Bubble Chamber Pictures Taken

1,517,772 pix

1. Pictures in Hydrogen $\left(\mathrm{H}_{2}\right)$

2. Pictures in Deuterium $\left(D_{2}\right)$
$1,340,362$ pix $177,410 \mathrm{pix}$

Total 30 inch Bubble Chamber Pictures Taken

$3,170,163 \mathrm{pix}$

1. Pictures in Hydrogen $\left(\mathrm{H}_{2}\right)$

2. Pictures in Deuterium $\left(D_{2}\right)$

3. Pictures in $\mathrm{H}_{2}+\mathrm{Ne}$

4. Pictures in $\mathrm{D}_{2}+\mathrm{Ne}$
1,$918 ; 113 \mathrm{pix}$ $1,139,051$ pix 48,433 pix 64,566 vix 
AGS OPERATIONS SUMMARY

January through June 1966

\begin{tabular}{|c|c|c|c|c|c|c|c|c|c|c|c|c|}
\hline \multirow[t]{2}{*}{ \% m } & \multicolumn{2}{|c|}{$\operatorname{Jan} 1-\operatorname{Jan} 25$} & \multicolumn{2}{|c|}{$\operatorname{Jan} 26-$ Feb 25} & \multicolumn{2}{|c|}{ Feb $26-\operatorname{Mar} 25$} & \multicolumn{2}{|c|}{ Mar 26 - Apr 25} & \multicolumn{2}{|c|}{ Apr 26 - May 25} & \multicolumn{2}{|c|}{ May 26 - Jun 20 } \\
\hline & Hours. & $\%$ & Hours & $\%$ & Hours & $0 \%$ & Hours & $0 / 0$ & Hours & $0 / 10$ & Hours & $0 \%$ \\
\hline w & 401.61 & 72.8 & 577.57 & 77.6 & 501.20 & 74.6 & 630.58 & 84.8 & 535.88 & 76.4 & 272.14 & 85.0 \\
\hline 1. For Experimenters & 341.45 & 61.9 & 514.50 & $69.1:$ & 446.68 & 66.5 & 556.81 & 74.9 & 472.39 & 65.6 & 240.35 & 75.1 \\
\hline 2. For Accelerator Research & 60.16 & 10.9 & 63.07 & 8.5 & 54.52 & 8.1 & 73.77 & 9.9 & 63.49 & 8.8 & 31.79 & 9.9 \\
\hline MACHINE DOWNTIME & 150.39 & 27.2 & 166.43 & 22.4 & 170.80 & 25.4 & 112.42 & 15.2 & 184.12 & 25.6 & 47.86 & 15.0 \\
\hline 1. Machine Set-up, Shutdown & 17.44 & 3.2 & 19.18 & 2.5 & 11.23 & 1.7 & iI. 78 & 1.6 & 19.82 & 2.7 & 5.04 & 1.6 \\
\hline 2. Requested Down & 4.90 & 0.9 & 3.00 & 0.4 & 4.12 & 0.6 & 5.80 & 0.8 & 5.74 & 0.8 & 1.33 & 0.4 \\
\hline 3. Experimental Set-up & 3.40 & 0.6 & 6.23 & 0.8 & 0.00 & 0.0 & 0.00 & 0.0 & 0.48 & 0.1 & 2.85 & 0.9 \\
\hline 4. Machine Failures & 69.22 & 12.5 & 64.69 & 8.7 & 115.77 & 17.2 & 65.53 & 8.8 & 108.57 & 15.0 & 30.29 & 9.5 \\
\hline 5. EAO Equipment Failures & 12.97 & 2.3 & 3.69 & 0.5 & 11.68 & 1.7 & 1.20 & 0.2 & 2.11 & 0.3 & 0.00 & 0.0 \\
\hline 6. Scheduled Maintenance & 42.46 & 7.7 & 69.64 & 9.4 & 28.00 & 4.2 & 28.11 & 3.8 & 47.40 & 6.7 & 8.35 & 2.6 \\
\hline SCHEDULED OPERATIONS & 552.00 & 100.0 & 744.00 & 100.0 & 672.00 & 100.0 & $743: 00$ & 100.0 & 720.00 & 200.0 & 320.00 & 100.0 \\
\hline HOLIDAYS & 48.00 & & & & & & & & & & & \\
\hline SHUTDOWN & & & & & & & & & & & 544.00 & \\
\hline AGS PERFORMANCE & & & & & & 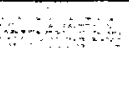 & & & \# & 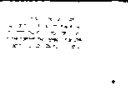 & & \\
\hline a. Magnet pulses & 644,691 & & 925,672 & & 765.156 & & 929,320 & & 895,981 & & 417,670 & \\
\hline b. Accelerated pulses & 518,696 & & $790,23]$ & & 661,031 & & $813,88 \%$ & & 687,740 & & 350,458 & \\
\hline c. Missed pulses & 41,303 & & 57,487 & & 44,338 & & 55,92 & & 63,911 & & 18,760 & \\
\hline d. Accelerated protons ( $p)$ & $4.2 \times$ & $10^{17}$ & 6.83 & $10^{-17}$ & $7.2=$ & $10^{17}$ & 9.82 & $10^{17}$ & 7.2 & $10^{17}$ & 3.7 & $10^{1} ?$ \\
\hline e. Aver $p /$ pulse $(d / b)$ & $8.1 \times$ & $10^{11}$ & 8.72 & $10^{11}$ & 10.92 & $10^{11}$ & $12.0=$ & $10^{11}$ & 10.5 & $10^{11}$ & 10.6 & $10^{11}$ \\
\hline f. Aver. p/pulse $(d / b+c)$ & $7.5 \times$ & $10^{11}$ & 8.1 & $10^{11}$ & $10.2 J$ & $10^{11}$ & 11.2 & $10^{11}$ & 9.6 & $10^{11}$ & 10.0 & $10^{11}$ \\
\hline
\end{tabular}


AGS OPERATIONS SUMMARY

July through December 1966






\section{AGS OPERATIONS SUMMARY}

Weekly

$\vdots$

January through March 1966

\begin{tabular}{|c|c|c|c|c|c|c|c|c|c|c|c|c|c|c|}
\hline \multirow{2}{*}{$\begin{array}{l}\text { Hours } \\
\text { Period }\end{array}$} & \multicolumn{4}{|c|}{ AGS ON } & \multicolumn{3}{|l|}{. } & \multicolumn{4}{|c|}{ AGS OFF } & \multirow{2}{*}{$\begin{array}{l}\text { Holi- } \\
\text { days }\end{array}$} & \multirow{2}{*}{$\begin{array}{l}\text { Shut- } \\
\text { down }\end{array}$} & \multirow{2}{*}{$\begin{array}{l}\text { Average } \\
\text { Intensity } \\
10^{11} \mathrm{p} / \mathrm{pul}\end{array}$} \\
\hline & $\begin{array}{l}\text { Sched.: } \\
\text { Oprs. } \\
\end{array}$ & HEP & $\begin{array}{c}\text { AGS } \\
\text { Stud. } \\
\end{array}$ & $\begin{array}{l}\text { Total } \\
\text { AGs On } \\
\end{array}$ & $\begin{array}{c}A G S \\
\text { Set-up } \\
\end{array}$ & $\begin{array}{l}\text { Req 'd' } \\
\text { Down }\end{array}$ & $\begin{array}{r}\text { Expt. } \\
\text { Set-up } \\
\end{array}$ & $\begin{array}{l}\text { Sched. } \\
\text { Maint. }\end{array}$ & $\begin{array}{r}\mathrm{EAO} \\
\mathrm{Fail} \\
\end{array}$ & $\begin{array}{l}\text { AGS } \\
\text { Fail. }\end{array}$ & $\begin{array}{l}\text { Total } \\
\text { Down }\end{array}$ & & & \\
\hline $1 / 1-1 / 5$ & 72.00 & 31.92 & 9.90 & 41.82 & 7.00 & 0.00 & 0.00 & 16.00 & 0.00 & 7.18 & 30.18 & 48.00 & $\because$ & $10.4 \% 9.1$ \\
\hline $1 / 6-1 / 12$ & 168.00 & 106.82 & 28.99 & 135.81 & 3.13 & 1.20 & 1.10 & 3.66 & 9.20 & 13.90 & 32.19 & & 8 & $9.0 / 8.2$ \\
\hline $1 / 13-1 / 19$ & 168.00 & 99.14 & 13.01 & 112.15 & 6.21 & 0.40 & 2.30 & 7.06 & 0.00 & 39.88 & 55.85 & 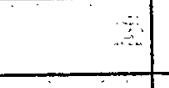 & & $8.0 / 7.5$ \\
\hline $1 / 20-1 / 26$ & 168.00 & 110.82 & 11.78 & 122.60 & 1.10 & 3.30 & 0.00 & 28.22 & 4.42 & 8.36 & 45.40 & & & $6.5 / 5.9$ \\
\hline $1 / 27-2 / 2$ & 168.00 & 98.51 & 7.42 & 105.93 & 7.45 & 0.41 & 3.30 & 30.90 & 1.34 & 18.67 & 62.07 & & & $8.2 / 7.7$ \\
\hline $2 / 3-2 / 9$ & 168.00 & 126.00 & 12.58 & 138.58 & 3.96 & 0.49 & 0.00 & 14.26 & 1.70 & 9.01 & 29.42 & & & $9.3 / 8.9$ \\
\hline $2 / 10-2 / 16$ & 168.00 & $\begin{array}{r}133.40 \\
\end{array}$ & 15.55 & 148.95 & 3.56 & 1.23 & 2.93 & 0,00 & 0.00 & 11.32 & 19.05 & & & $8.3 / 7.6$ \\
\hline $2 / 17-2 / 23$ & 168.00 & 107.29 & 24.00 & 131.39 & 1.60 & 0.00 & 0.00 & 12.00 & 0.00 & 23.01 & 36.61 & $\because$ & 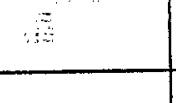 & $8.7 / 8.0$ \\
\hline $2 / 24-3 / 2$ & 168.00 & 142.07 & 14.84 & 156.91 & 2.81 & 0.87 & 0.00 & 0.00 & 0.94 & 6.47 & 11.09 & $\therefore$ & . & $9.5 \% 9.0$ \\
\hline $3 / 3-3 / 9$ & 168.00 & 112.65 & 21.95 & 134.60 & 5.10 & 0.25 & 0.00 & 16.00 & 3.20 & 8.85 & 33.40 & & & $9.4 / 8.8$ \\
\hline $3 / 10-3 / 16$ & 168.00 & 128.55 & 0.98 & 129.53 & 0.64 & 2.45 & 0.00 & 12.00 & 2.03 & 21.35 & 38.47 & + & & $11.9 / 11.5$ \\
\hline $3 / 17-3 / 23$ & 168.00 & 67.15 & 16.10 & 83.25 & 4.24 & 1.16 & 0.00 & 0.00 & 5.51 & 73.84 & 84.75 & & & $13.0 / 11.8$ \\
\hline $3 / 24-3 / 30$ & 168.00 & 139.24 & 12.98 & 152.22 & 1.55 & 1.01 & 0.00 & 0.00 & 0.00 & 13.22 & 15.78 & & & $11.4 / 10.5$ \\
\hline Totals, & 2088.00 & 1403.66 & 190.08 & 1593.74 & 48.35 & 12.77 & 9.63 & 140.10 & 28.34 & 255.07 & 494.26 & 48.00 & 0.00 & $9.4 / 8.7$ \\
\hline $0 \%$ & 100.0 & 67.2 & 9.1 & 76.3 & 2.3 & 0.6 & 0.5 & 6.7 & 1.4 & 12.2 & 23.7 & & 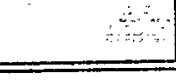 & - \\
\hline
\end{tabular}


AGS OPERATIONS SUMMARY.

Weekly

April through June 1966

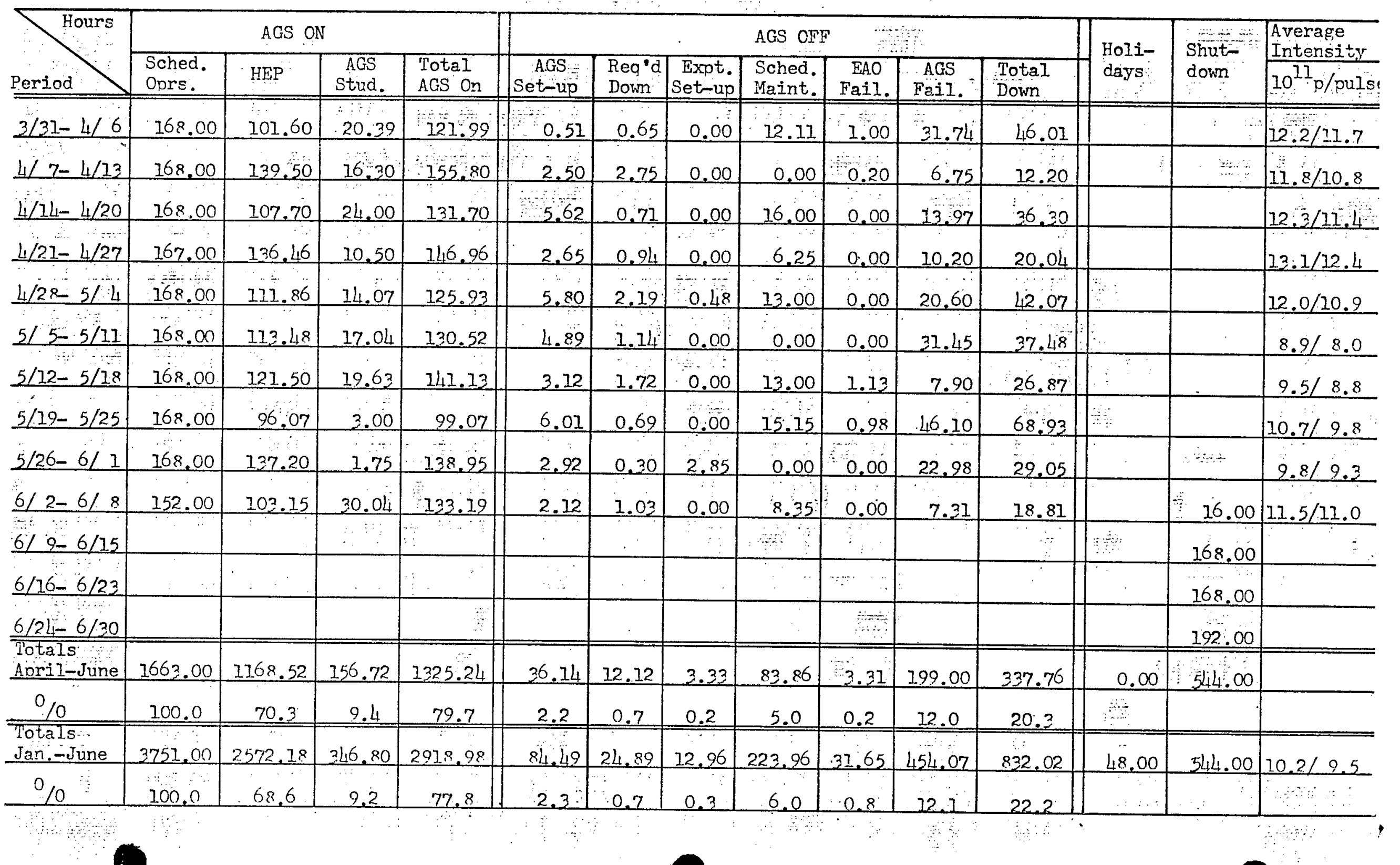




\section{Weekly}

July through September 1966

\begin{tabular}{|c|c|c|c|c|c|c|c|c|c|c|c|c|c|c|}
\hline \multirow[b]{2}{*}{ Period } & \multicolumn{3}{|c|}{ AGS ON } & 4 & \multicolumn{5}{|c|}{ - $A G S O F F$} & \multicolumn{2}{|c|}{$6=6+4$} & \multirow{2}{*}{$\begin{array}{l}\text { Holi- } \\
\text { days }\end{array}$} & \multirow{2}{*}{$\begin{array}{l}\text { Shut- } \\
\text { down }\end{array}$} & \multirow{2}{*}{$\begin{array}{l}\text { Average } \\
\text { Intensity } \\
10^{11} \mathrm{p} / \text { puls } \\
\end{array}$} \\
\hline & $\begin{array}{l}\text { Sched. } \\
\text { Oprs. }\end{array}$ & HEP & $\begin{array}{r}\text { AGS } \\
\text { Stud. } \\
\end{array}$ & $\begin{array}{l}\text { Total } \\
\text { AGS On }\end{array}$ & $\begin{array}{c}\text { AGS } \\
\text { Set-up } \\
\end{array}$ & $\begin{array}{l}\text { Req } \mathrm{d} \\
\text { Down }\end{array}$ & $\begin{array}{r}\text { Expt. } \\
\text { Set-up } \\
\end{array}$ & $\begin{array}{l}\text { Sched: } \\
\text { Maint: }\end{array}$ & $\begin{array}{r}\mathrm{EAO} \\
\text { Fai1 } \\
\end{array}$ & $\begin{array}{c}\text { AGS } \\
\text { Fail. } \\
\end{array}$ & $\begin{array}{l}\text { Total } \\
\text { Down } \\
\end{array}$ & & & \\
\hline $7 / 1-7 / 6$ & & & & $\therefore$ & $\therefore$ & & & 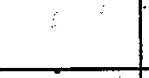 & 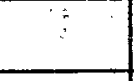 & & & 48.00 & 96.00 & \\
\hline $7 / 7-7 / 13$ & 72.00 & 0.00 & 0.00 & 0.00 & 42.80 & 0.00 & 0.00 & 29.20 & 0.00 & 0.00 & 72.00 & & 96.00 & 1.91 \\
\hline $7 / 14-7 / 20$ & 168.00 & 70.38 & 34.08 & 104.46 & 5.47 & 3.92 & 2.35 & 15.27 & 21.95 & 14.64 & 63.54 & & & $10.6 / 9.1$ \\
\hline $7 / 21-7 / 27$ & 168.00 & 106.84 & 10.63 & 117.47 & 10.35 & 0.00 & 2.35 & 24.00 & 0.00 & 13.82 & 50.53 & & 8 & $12.6 / 11.4$ \\
\hline $7 / 28-8 / 3$ & 168.00 & 121.48 & 12.55 & 134.03 & 2.75 & 2.85 & 2.15 & 0.00 & 1.75 & 24.47 & 33.97 & & & $14.6 / 13.5$ \\
\hline $8 / 4-8 / 10$ & 168.00 & 110.55 & 15.22 & 125.77 & 7.29 & 2.10 & 0.75 & 19.66 & 0.00 & 12.43 & 42.23 & & & $13.8 / 12.7$ \\
\hline $8 / 11-8 / 17$ & 168.00 & 98.69 & 13.40 & 112.09 & $\quad 2,83$ & 0.93 & 2.27 & 0.00 & 0.00 & 48.88 & 55.91 & & & $13.8 / 12.6$ \\
\hline $8 / 18-8 / 24$ & 168.00 & 126.14 & 7.64 & 133.78 & 3.92 & 0.65 & 0.00 & 14.00 & 0.55 & 15.10 & 34.22 & & & $13.9 / 13.0$ \\
\hline $8 / 25-8 / 31$ & 168.00 & 127.99 & 13.83 & 141.82 & 2.95 & 1.57 & 0.00 & 16.00 & 0.00 & 5.66 & 26.18 & & & $16.5 / 15.6$ \\
\hline $9 / 1-9 / 7$ & 168.00 & 96.59 & 16.62 & 113.21 & 16.81 & 0.00 & 0.00 & 1.15 & 0.00 & 36.83 & 54.79 & & & $11.2 / 10.4$ \\
\hline $9 / 8-9 / 14$ & 168.00 & 124.91 & 31.78 & 156.69 & 1.07 & 0.92 & 0.00 & 0.00 & 1.62 & 7.70 & 11.31 & & & $14.7 / 13.9$ \\
\hline $9 / 15-9 / 21$ & 168.00 & $\begin{array}{r}\vdots \\
90.30 \\
\end{array}$ & 12.26 & 102.56 & 7.47 & 0.00 & 2.00 & 27.60 & 0.75 & 37.62 & 65.44 & & & $14.3 / 13.3$ \\
\hline $9 / 22-9 / 28$ & 168.00 & 70.42 & 8.85 & 79.27 & 5.08 & 7.31 & 0.75 & 64.00 & 0.00 & 11.59 & 88.73 & & & $11.2 / 10.1$ \\
\hline Totals & 1920.00 & 3144.29 & 176.86 & 1321.15 & 109.73 & 20.25 & 12.62 & 200.88 & 26.62 & 228.75 & 598.85 & 48.00 & 192.00 & 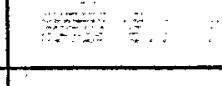 \\
\hline $0 \%$ & 100.0 & 59.6 & 9.2 & 68.8 & 5.7 & 1.1 & 0.6 & 10.5 & 1.4 & 11.9 & 31.2 & & & \\
\hline
\end{tabular}


AGS OPERATIONS SUMMARY

Weekly

October through December 1966

\begin{tabular}{|c|c|c|c|c|c|c|c|c|c|c|c|c|c|c|}
\hline \multirow{2}{*}{$\begin{array}{l}\text { Hours } \\
\text { Period }\end{array}$} & \multicolumn{4}{|c|}{ AGS ON } & \multicolumn{7}{|c|}{ AGS OFF } & \multirow{2}{*}{$\begin{array}{l}\text { Holi- } \\
\text { days }\end{array}$} & \multirow{2}{*}{$\begin{array}{l}\text { Shut- } \\
\text { down }\end{array}$} & \multirow{2}{*}{$\begin{array}{l}\text { Average } \\
\text { Intensity } \\
10^{11} \mathrm{p} / \mathrm{puls} \\
\end{array}$} \\
\hline & $\begin{array}{l}\text { Sched. } \\
\text { Onrs: }\end{array}$ & $H E P$ & $\begin{array}{r}\text { AGS } \\
\text { Stud. } \\
\end{array}$ & $\begin{array}{l}\text { Totai } \\
\text { AGS On } \\
\end{array}$ & $\begin{array}{l}\text { AGS } \\
\text { Set-up } \\
\end{array}$ & $\begin{array}{l}\text { Reg }{ }^{\circ} \\
\text { Down }\end{array}$ & \begin{tabular}{|r|} 
Expt: \\
Set-up \\
\end{tabular} & $\begin{array}{l}\text { Sched. } \\
\text { Maint. }\end{array}$ & $\begin{array}{r}\text { EAO } \\
\text { Fail. } \\
\end{array}$ & $\begin{array}{r}\text { AGS } \\
\text { Fail. }\end{array}$ & $\begin{array}{l}\text { Total } \\
\text { Down } \\
\end{array}$ & & & \\
\hline $9 / 29-10 / 5$ & 168.00 & 132.01 & 15.69 & 147.70 & 9.17 & 1.98 & $1: 20$ & 0.42 & 2.06 & 5.47 & 20.30 & & 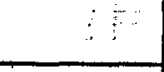 & $13.4 / 12.4$ \\
\hline $10 / 6-10 / 12$ & 168.00 & 139.89 & 13.10 & 152.99 & 2.75 & 1.50 & 0.00 & 2.20 & 0.00 & 8.56 & 15.01 & & 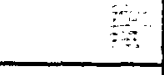 & $14.7 / 14.0$ \\
\hline $10 / 12=10 / 19$ & 168.00 & 1.14 .47 & 11.96 & 129.42 & 4.90 & 0.00 & 0.00 & 13.58 & 1.05 & 19.04 & 38.57 & & & $14.1 / 13.4$ \\
\hline $10 / 27-11 / 2$ & -169.00 & 107.31 & 1.5 .89 & 123.20 & 9.03 & 0.00 & 0.00 & 20.07 & 0.00 & 16.70 & 45.80 & & $\because$ & $14.5 / 14.0$ \\
\hline $11 / 3-11 / 9$ & 168.00 & 141.18 & 2.60 & 143.78 & 2.40 & 0.89 & 0.00 & 16,00 & 0.00 & 4.93 & 24.22 & & & $10.6 / 10.2$ \\
\hline $11 / 10-11 / 16$ & 168.00 & 101.75 & 37.43 & 138.18 & 3.47 & 0.00 & 3.32 & 9.00 & 3.20 & 9.82 & 28.82 & & $=$ & $9.9 / 9.1$ \\
\hline $11 / 17-11 / 23$ & 168.00 & 100.40 & 11.20 & 111.60 & 4.50 & 2.52 & 0.15 & 23.50 & 0.00 & 25.73 & 56.40 & & & $11.7 / 10.5$ \\
\hline $11 / 24-11 / 30$ & 104.00 & 92.55 & 0.80 & 93.35 & 1.85 & 0.00 & 0.00 & 0.00 & 0.75 & 8.05 & 10.65 & & 64.00 & $16.1 / 15.6$ \\
\hline $12 / 1-12 / 7$ & 136.00 & $\begin{array}{r}83.36 \\
\end{array}$ & 9.30 & 92.66 & 7.96 & 1.54 & 0.00 & 8.30 & 2.96 & 22.58 & 43.34 & & 32.00 & $14.5 / 13.7$ \\
\hline $12 / 8-12 / 14$ & 40.00 & 33.54 & 0.26 & 32.70 & 1.20 & 2.00 & 0.00 & 0.00 & 1.85 & 1.25 & 6.30 & & 128.00 & $13.3 / 13.0$ \\
\hline $12 / 15-12 / 21$ & 160.00 & 100.03 & 8.30 & 108.33 & 19.68 & 1.80 & 0.00 & 8.00 & 5.18 & 17.01 & 51.67 & $\therefore$ & 8.00 & $9.6 / 9.1$ \\
\hline$\frac{12 / 22-12 / 21}{\text { Tot } 15}$ & 28.00 & 8.00 & 8.92 & 16.92 & $\begin{array}{r}10.15 \\
\end{array}$ & 0.00 & 0.00 & 0.00 & 0.00 & 0.93 & 11.08 & 116.00 & 96.00 & $9.8 / 8.2$ \\
\hline $\begin{array}{l}\text { Lota } 15 \\
\text { Lth auarter }\end{array}$ & 1813.00 & 3.274 .80 & 156.96 & 1431.76 & 78.82 & 13.03 & 6.17 & 115.07 & 17.75 & 150.40 & 381.24 & 116.00 & 328.00 & \\
\hline $0 / 0$ & 100.0 & 70.3 & 8.7 & 79.0 & i.? & 0.7 & 0.3 & 6.4 & 1.0 & 8.3 & 21.0 & & & \\
\hline $\begin{array}{l}\text { Totals } \\
\text { July-Dec }\end{array}$ & 3732.00 & $2 l_{119.09}$ & 233.82 & 2752.91 & 188.55 & 32.28 & 18.79 & 315.95 & 44.37 & 379.15 & 980.09 & 16400 & 520.00 & $13.2 / 12.2$ \\
\hline $0 \%$ & 100.0 & 64.8 & 82 & 73.7 & 5.0 & 0.9 & 0.5 & 8.5 & 1.2 & 10.2 & 26.3 & 3 & & \\
\hline
\end{tabular}


AGS ON for High Energy Physics and Yachine Studies

Hours per Week

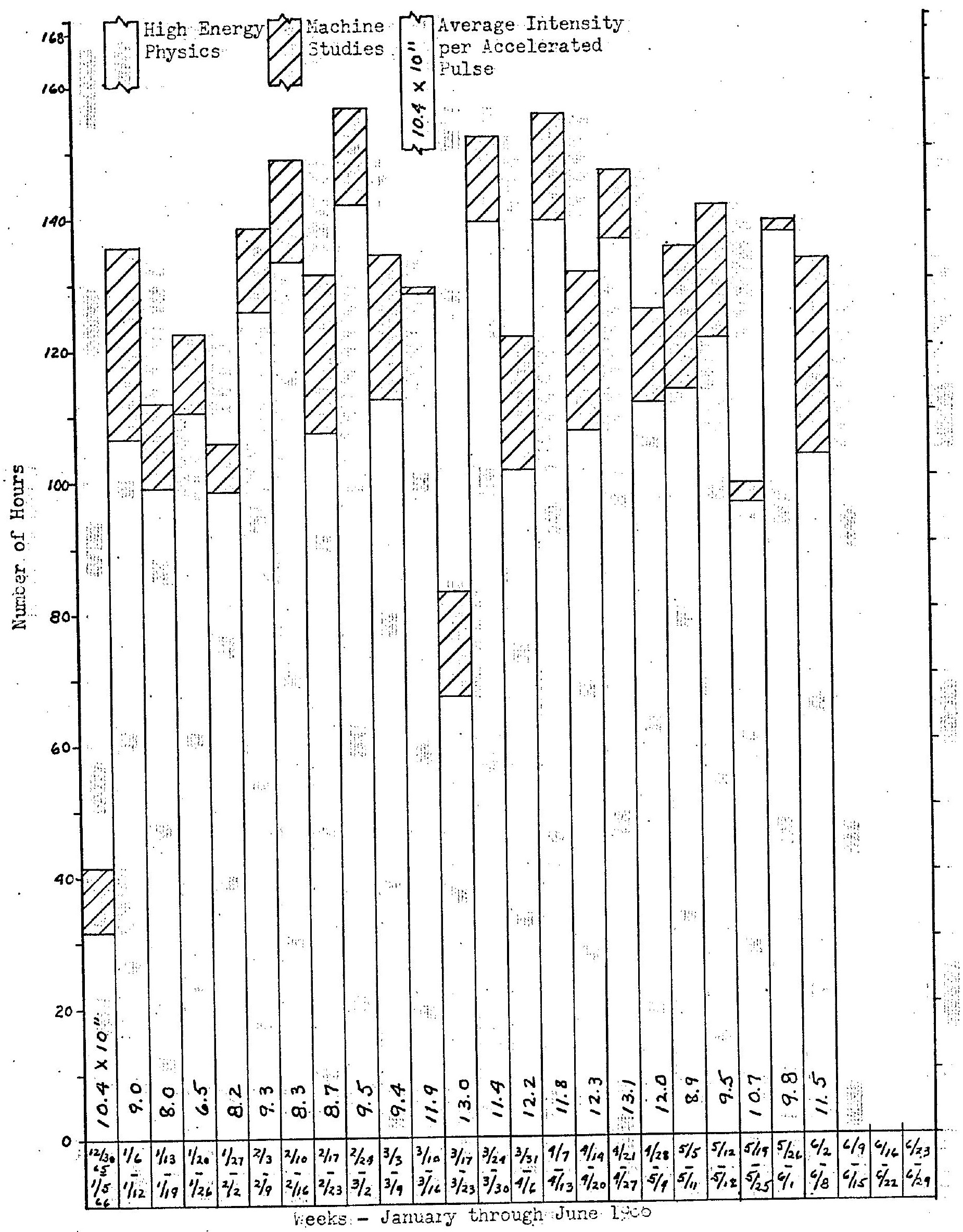


AGS ON for High Energy. Physics and Machine Studies

Hours per Week

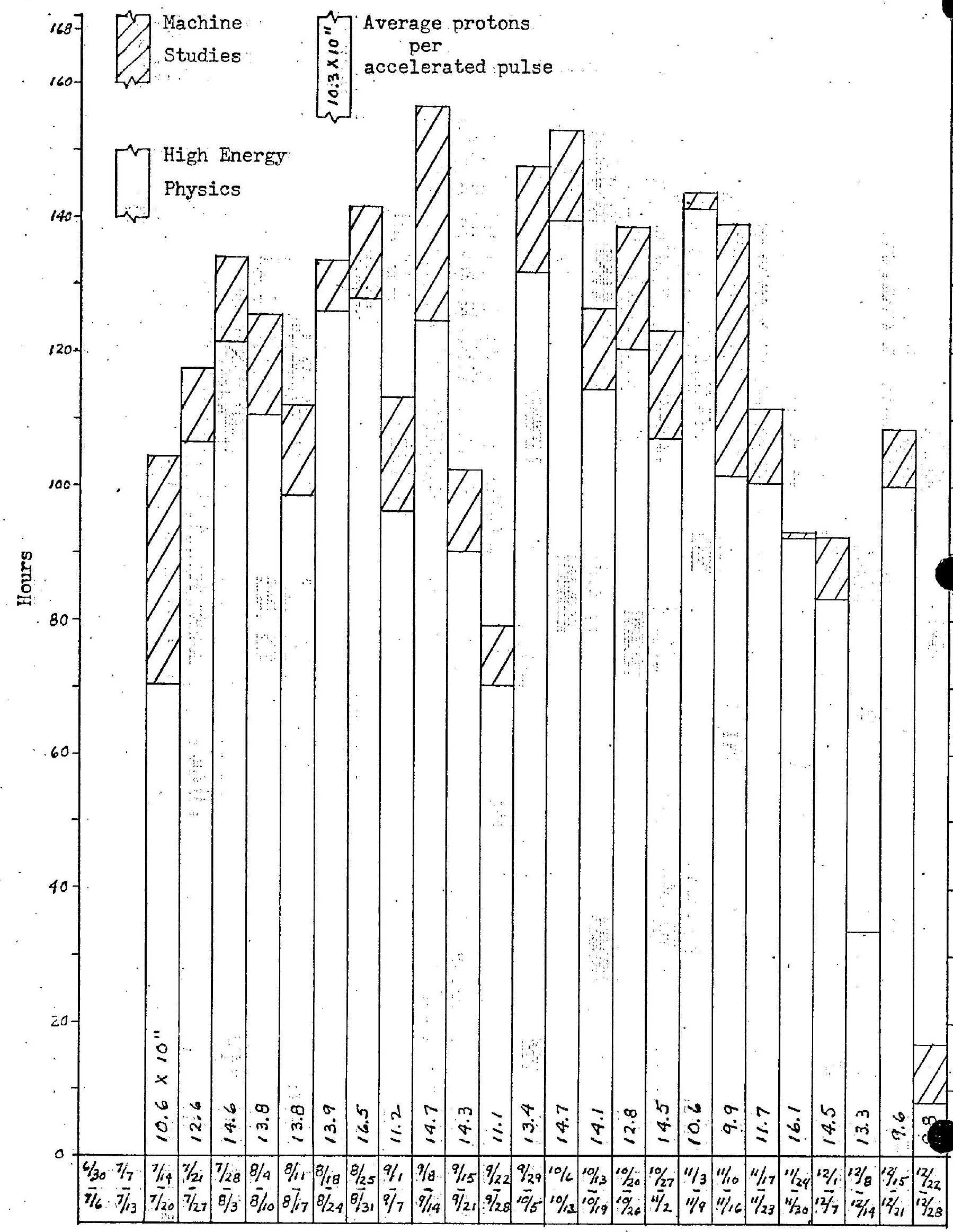

Weeks - July through December 1966 


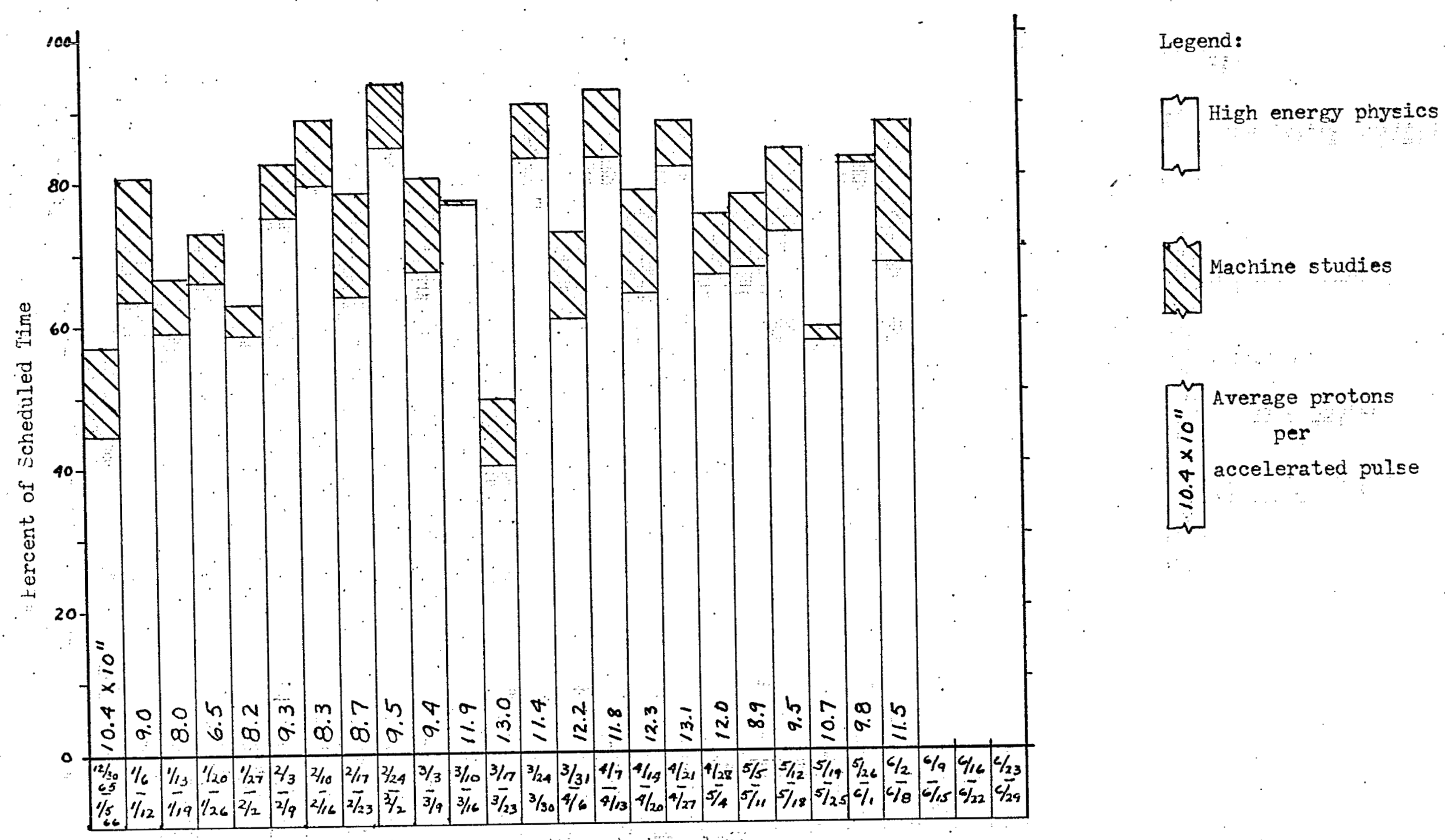




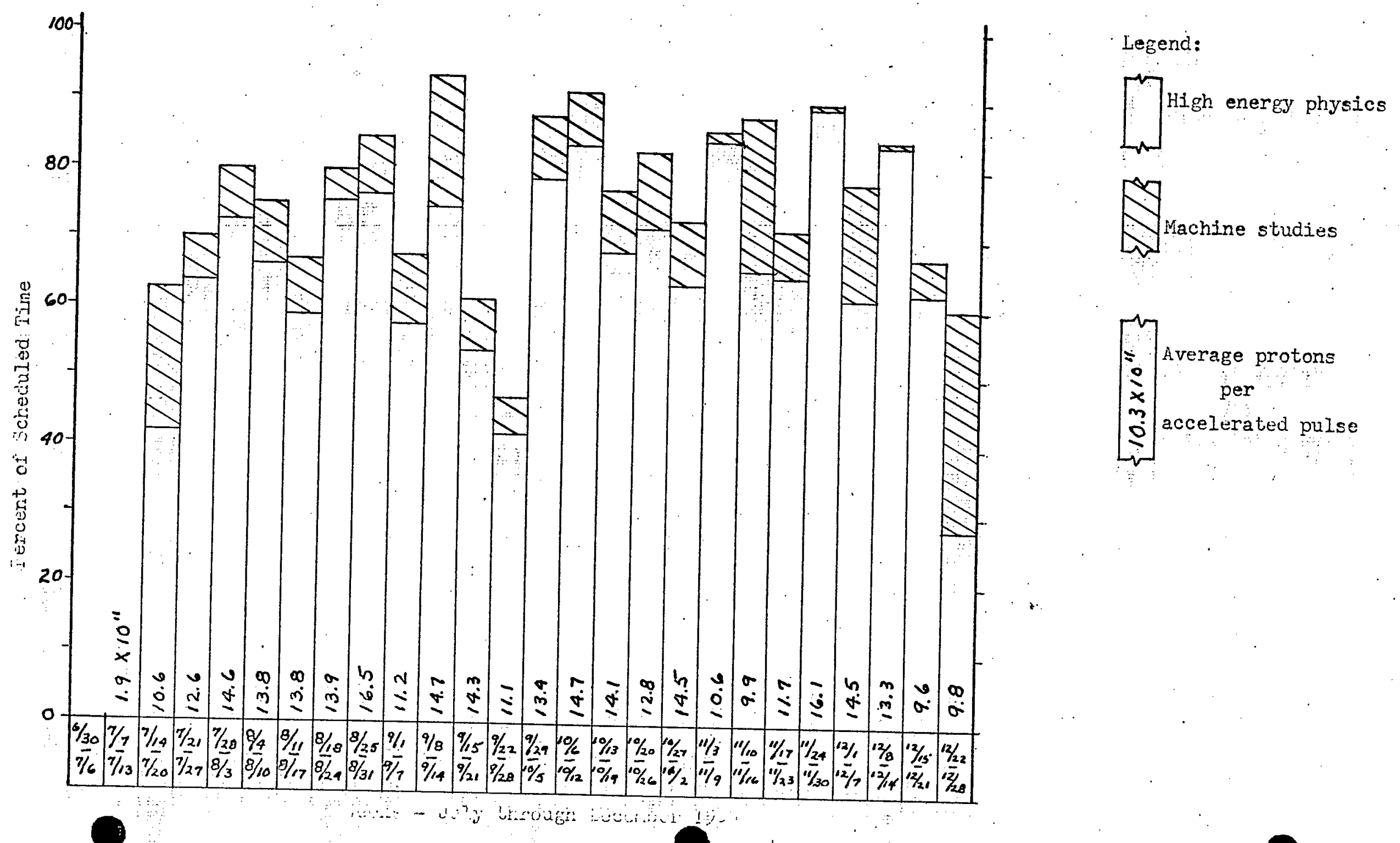


MACHINE DOWNTIME

percent of Scheculed lime

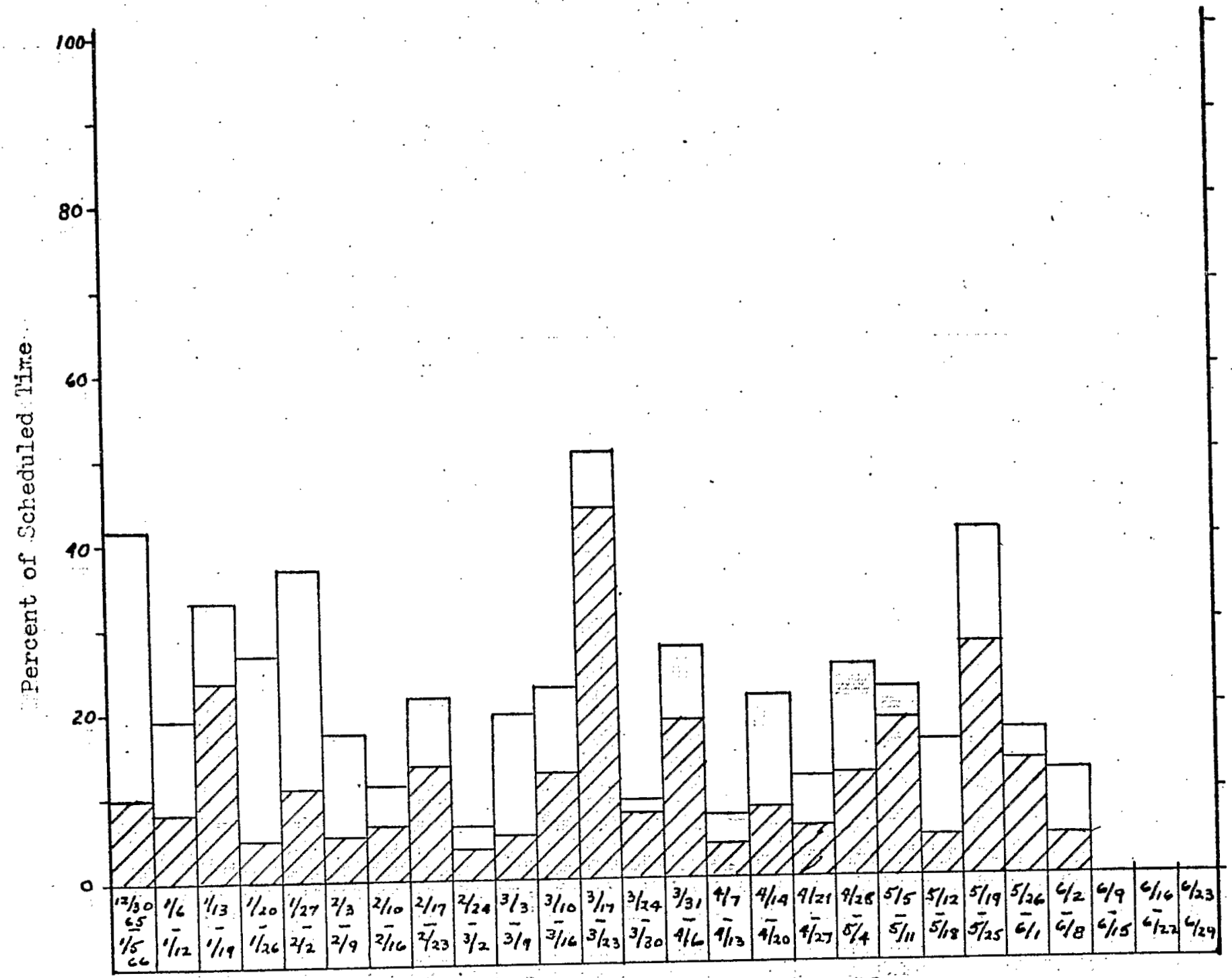

Hede - Janwary through Jime 1900
Legend:

$\square$

All downtime

except machine failures

$\theta$

Machine fallures 
MCHINE LU, HWE

rercent of Scheduled lizme

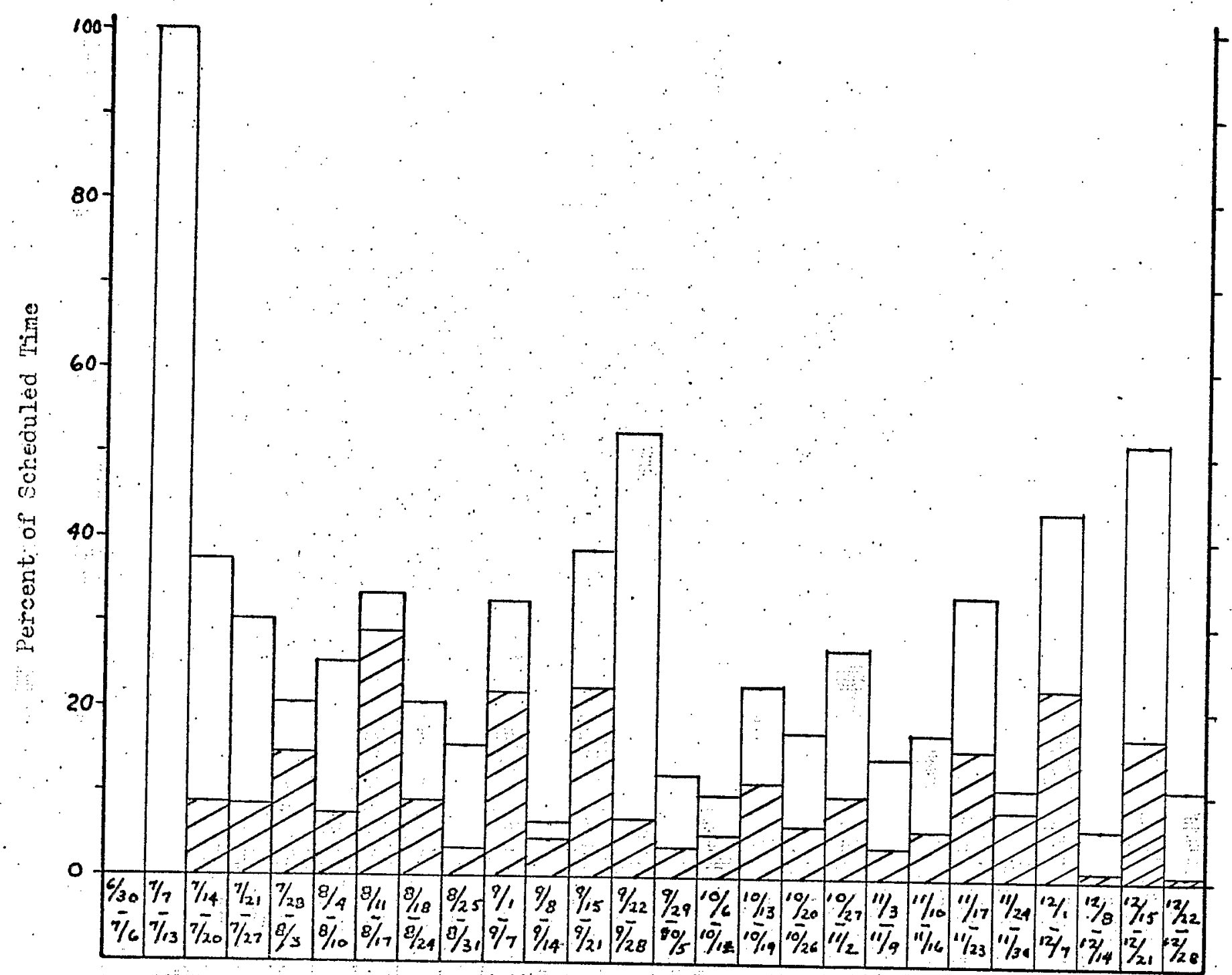

Legend:

7 All downtime

except

machine iailures

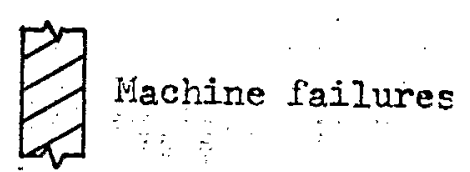








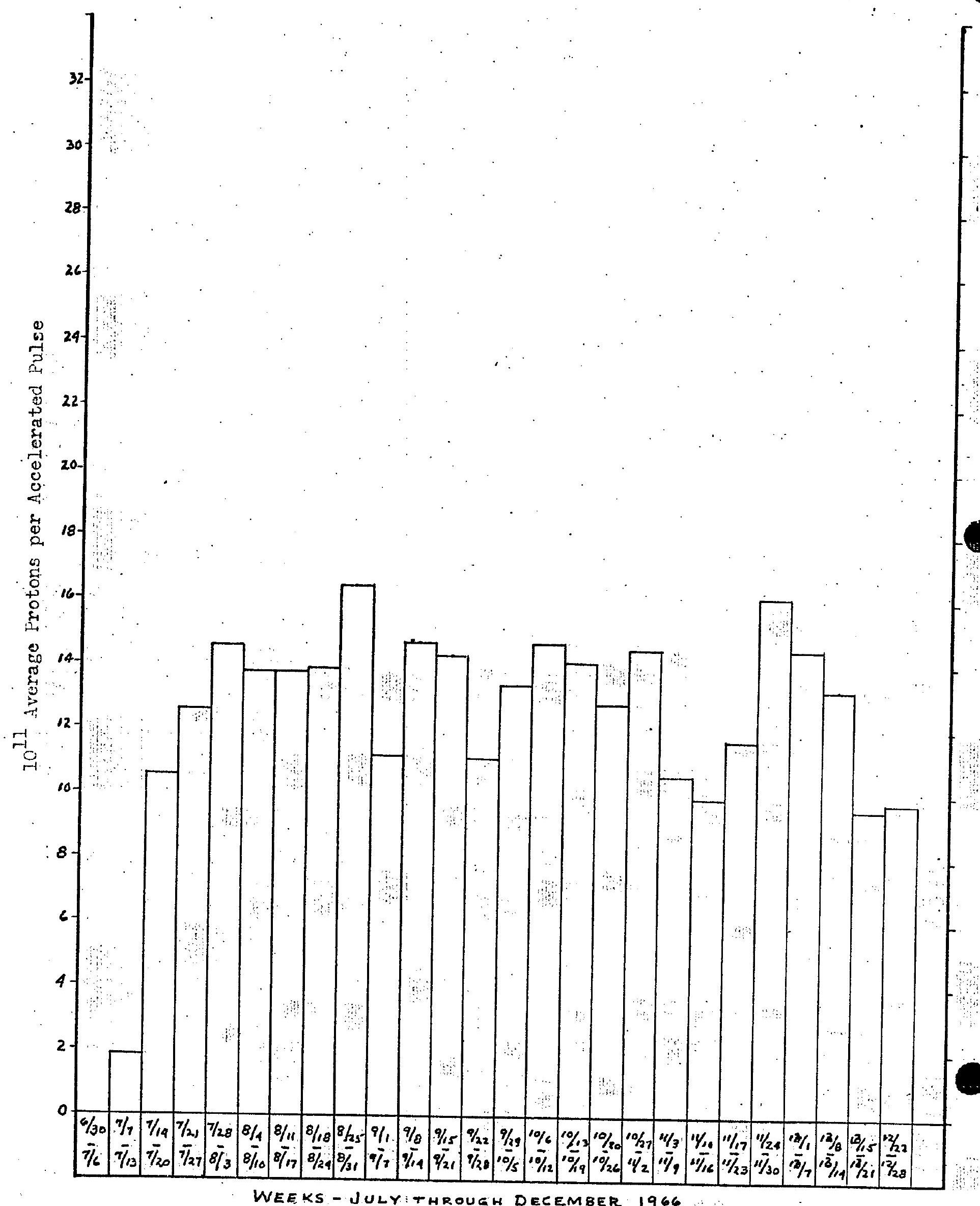
Experimental Users ' Operations Summary

30 inch Bubble Chamber Experiments

January through June 1966

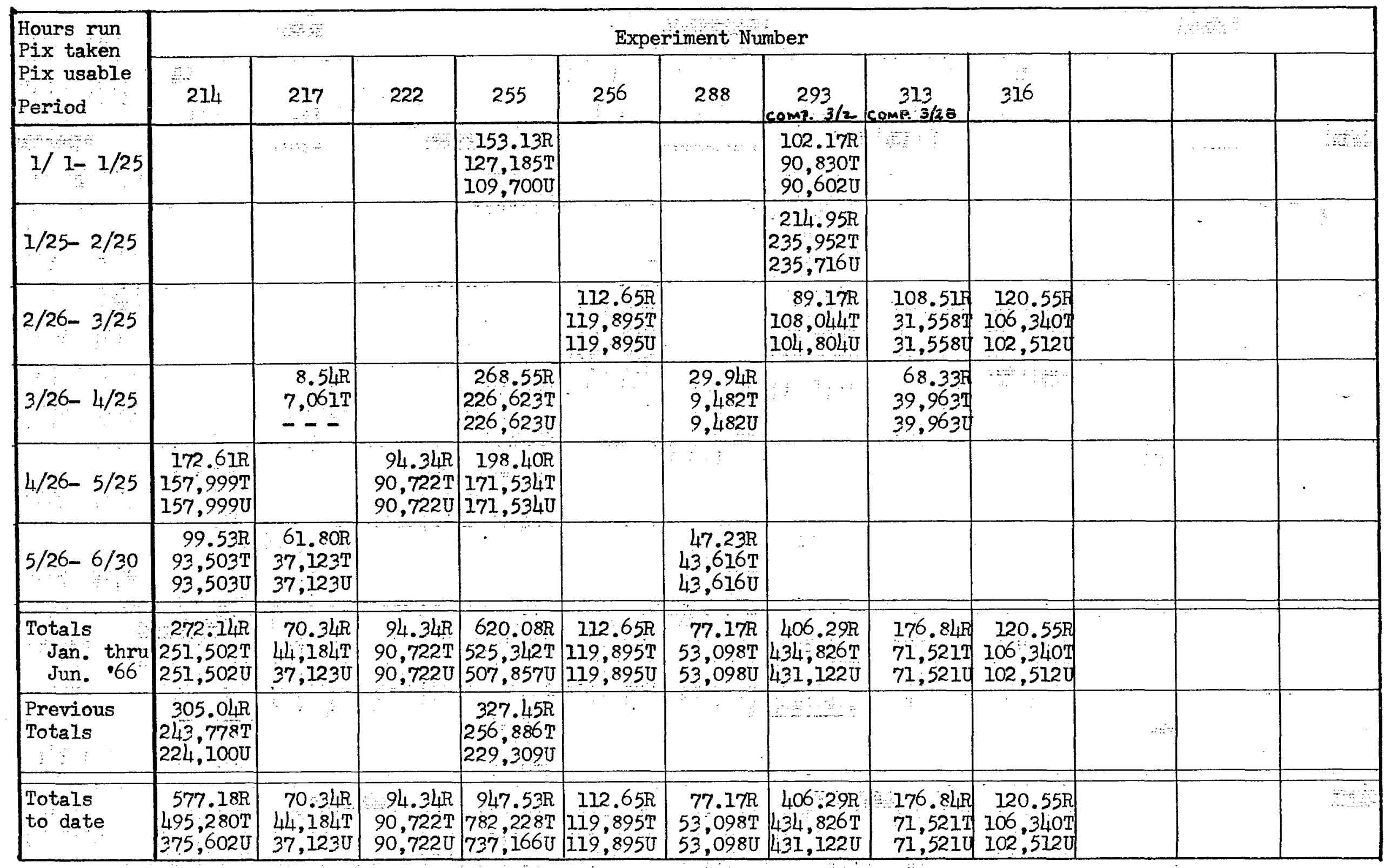


Experimental Users' Operations Summary

30 inch Bubble Chamber Experiments

July through December 1966

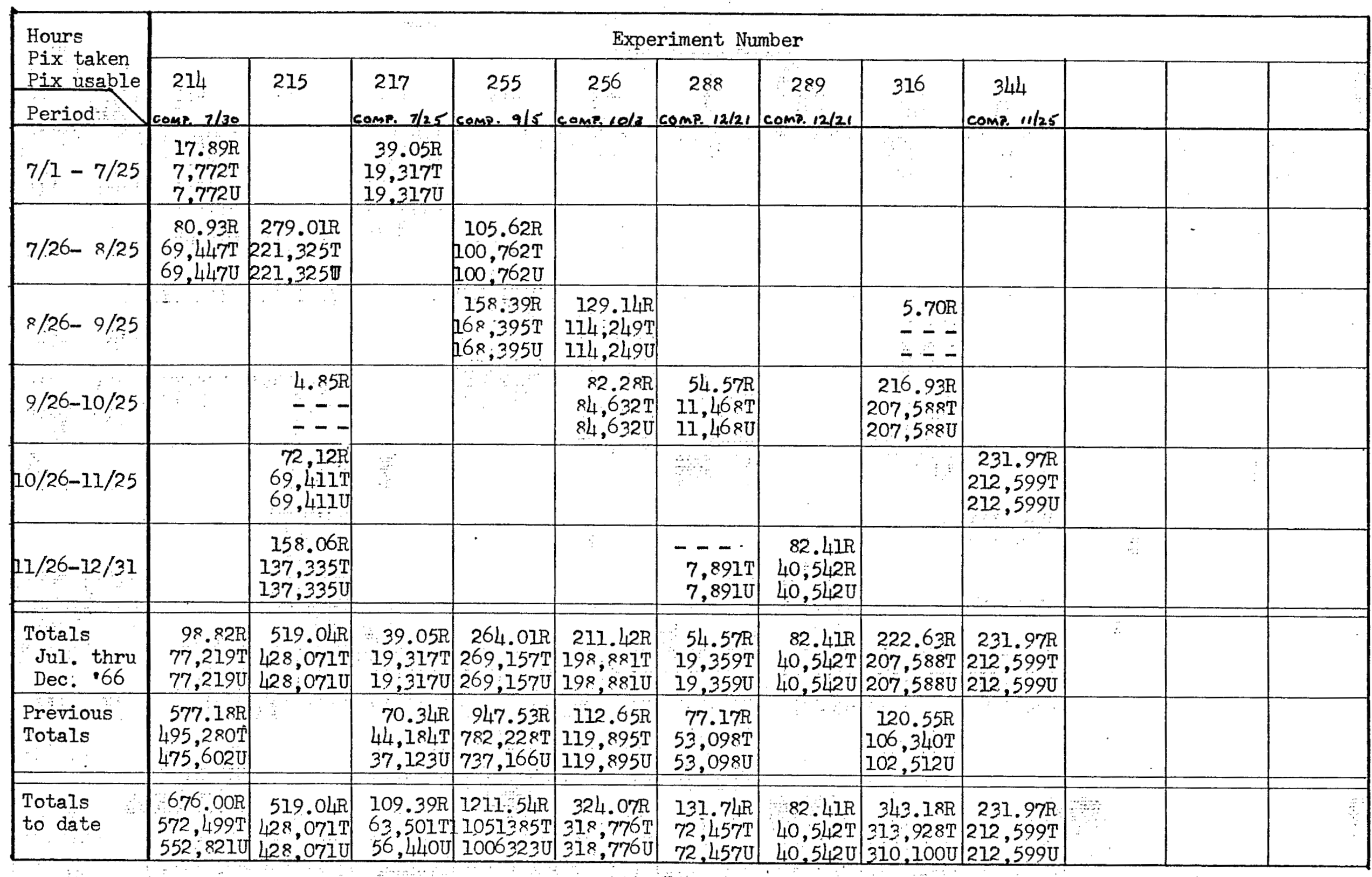


Experimental Users" Operations Summary

80 inch Bubble Chamber Experiments

January through June 1966

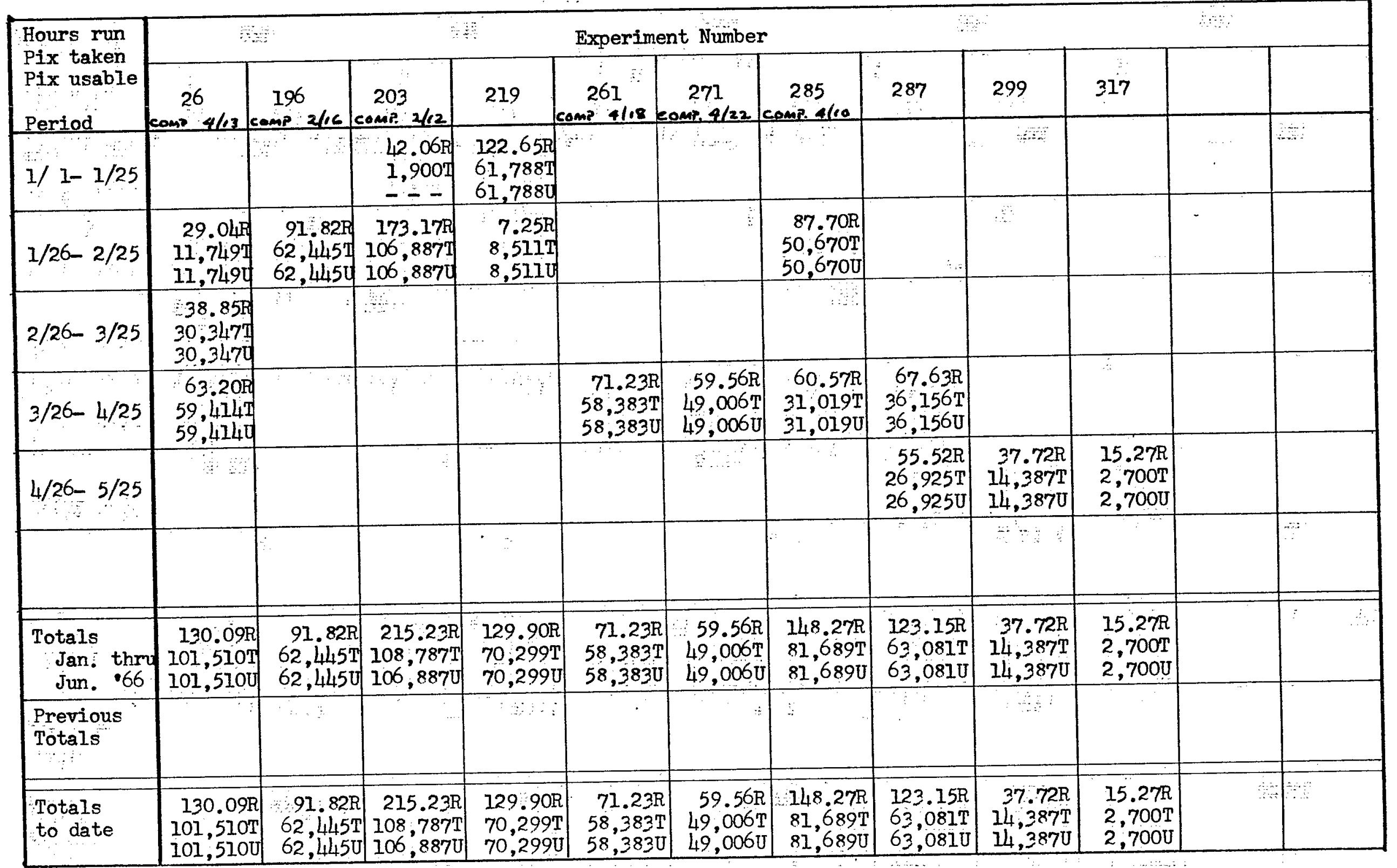


Experimental Users ' Operations Summary

80 inch Bubble Chamber Experiments

July through December 1966

\begin{tabular}{|c|c|c|c|c|c|c|c|c|c|c|c|c|c|c|c|}
\hline $\begin{array}{l}\text { Hours Run } \\
\text { Pix Taken }\end{array}$ & & & & & & & Experime & ent Number & & & & & $\because$ & & $\vdots$ \\
\hline Period & 203 & $\begin{array}{c}216 \\
\text { comp. rotar }\end{array}$ & $\begin{array}{c}219 \\
\text { comr } / 1 / 9\end{array}$ & 239 & $\begin{array}{c}251 \\
\text { comp. Ill }\end{array}$ & $\begin{array}{c}267 \\
\text { comr. } 11 / 7\end{array}$ & $\begin{array}{c}299 \\
\text { comp 8/25 }\end{array}$ & $\begin{array}{c}317 \\
\text { comp.10/12 }\end{array}$ & $\begin{array}{c}319 \\
\operatorname{comp~} 10 / 2\end{array}$ & $\begin{array}{c}328 \\
\text { comp } 11(20\end{array}$ & 335 & 341 & $\begin{array}{c}343 \\
\text { comp } 8 / 30\end{array}$ & ' & \\
\hline $7 / 1-7 / 25$ & $\begin{array}{l}120.4 \mathrm{IR} \\
32,468 \mathrm{~T} \\
32,468 \mathrm{U}\end{array}$ & & : & & $\therefore$ & & & \begin{tabular}{cc|}
$\vdots$ & $\vdots$ \\
$\vdots$ & \\
\end{tabular} & & & . & & & & \\
\hline $7 / 26-8 / 25$ & $\begin{array}{r}72.91 \mathrm{R} \\
52,033 \mathrm{~T} \\
52,033 \mathrm{U}\end{array}$ & & & & & & \begin{tabular}{|r|}
$210,39 R$ \\
$106,474 \mathrm{~T}$ \\
82,4740 \\
\end{tabular} & & & & & & - & & · \\
\hline $8 / 26-9 / 25$ & . & 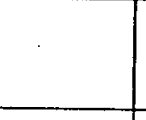 & & & & & & & $\begin{array}{r}68.57 \mathrm{R} \\
52,423 \mathrm{~T} \\
52,423 \mathrm{U} \\
\end{array}$ & & & $\begin{array}{l}30.291 \\
3,3521 \\
3,3524\end{array}$ & $\begin{array}{r}85,398 \\
53,1971 \\
53,1974 \\
\end{array}$ & & \\
\hline $9 / 26-10 / 25$ & . & $\begin{array}{l}115.92 \mathrm{R} \\
86,939 \mathrm{~T} \\
86 ; 939 \mathrm{U}\end{array}$ & & & & & & $\begin{array}{r}185,59 \mathrm{R} \\
128,180 \mathrm{~T} \\
108,180 \mathrm{U} \\
\end{array}$ & $\begin{array}{r}70.34 \mathrm{R} \\
59,986 \mathrm{~T} \\
59,986 \mathrm{U} \\
\end{array}$ & & $\begin{array}{r}92,267 \\
63,4271 \\
63,4274 \\
\end{array}$ & & & & \\
\hline $\begin{array}{c}10 / 26-11 / 25 \\
:\end{array}$ & & & $\begin{array}{r}31.40 \mathrm{R} \\
30,762 \mathrm{~T} \\
30,762 \mathrm{U}\end{array}$ & $\begin{array}{r}63,81 \mathrm{R} \\
17,400 \mathrm{~T} \\
17,400 \mathrm{U}\end{array}$ & $\begin{array}{r}91,96 \mathrm{R} \\
76,487 \mathrm{~T} \\
76,487 \mathrm{U}\end{array}$ & $\begin{array}{r}95.03 \mathrm{R} \\
72,823 \mathrm{~T} \\
72,823 \mathrm{U}\end{array}$ & & $\vdots \quad \vdots$ & - & $\begin{array}{l}114.111 \\
53,8291 \\
53,8294\end{array}$ & $\therefore \begin{array}{lll} & \therefore \\
\because & & \end{array}$ & & & & \\
\hline $11 / 26-12 / 31$ & & & & $\begin{array}{r}37.85 \mathrm{R} \\
15,705 \mathrm{~T} \\
15,705 \mathrm{U} \\
\end{array}$ & & & & & 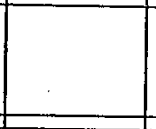 & & & & & & \\
\hline \begin{tabular}{|c|} 
Totals \\
Jul. thru \\
Dec. 66
\end{tabular} \mid & $\begin{array}{l}193.32 \mathrm{R} \\
84,501 \mathrm{~T} \\
84,501 \mathrm{U} \\
215.23 \mathrm{R}\end{array}$ & $\begin{array}{l}115.92 \mathrm{R} \\
86,939 \mathrm{~T} \\
86,9390\end{array}$ & $\begin{array}{r}31.40 \mathrm{R} \\
30,762 \mathrm{~T} \\
30,762 \mathrm{~J}\end{array}$ & $\begin{array}{l}119,26 \mathrm{R} \\
33,105 \mathrm{~T} \\
33,105 \mathrm{U}\end{array}$ & $\begin{array}{r}=91,96 \mathrm{R} \\
76,487 \mathrm{~T} \\
76,4870 \\
\end{array}$ & $\begin{array}{r}95.03 \mathrm{R} \\
72 ; 823 \mathrm{~T} \\
72 ; 823 \mathrm{U} \\
\end{array}$ & \begin{tabular}{r|}
$210,39 \mathrm{R}$ \\
$106,474 \mathrm{~T}$ \\
$82,474 \mathrm{U}$ \\
\end{tabular} & \begin{tabular}{|}
$185.59 \mathrm{R}$ \\
$128,180 \mathrm{~T}$ \\
$108,180 \mathrm{U}$ \\
\end{tabular} & $\begin{array}{r}138.91 R \\
112 ; 409 \mathrm{~T} \\
112 ; 409 \mathrm{U}\end{array}$ & $\begin{array}{l}114,119 \\
53,8291 \\
53,8294\end{array}$ & $\begin{array}{r}92,26 \mathrm{~F} \\
63,4271 \\
63,4274 \\
\end{array}$ & $\begin{array}{l}30.29 \mathrm{R} \\
3,3521 \\
3,3524\end{array}$ & $\begin{array}{r}85,391 \\
53,1979 \\
53,1970 \\
\end{array}$ & & , \\
\hline $\begin{array}{l}\text { Previous } \\
\text { Totals }\end{array}$ & $\begin{array}{r}215.23 \mathrm{R} \\
108,787 \mathrm{~T} \\
106,887 \mathrm{U}\end{array}$ & & $\begin{array}{l}129,90 \mathrm{R} \\
70,299 \mathrm{~T} \\
70,299 \mathrm{U}\end{array}$ & & & : & $\begin{array}{r}37.72 \mathrm{R} \\
14,387 \mathrm{~T} \\
14,387 \mathrm{U} \\
\end{array}$ & $\begin{array}{l}15: 27 \mathrm{R} \\
2,700 \mathrm{~T} \\
2,700 \mathrm{U} \\
\end{array}$ & . & $\cdots \cdots$ & : & & $: \quad:$ & & \\
\hline $\begin{array}{l}\text { Totals } \\
\text { to date }\end{array}$ & $\begin{array}{r}408,55 \mathrm{R} \\
193,288 \mathrm{~T} \\
191,388 \mathrm{U} \\
\end{array}$ & $\begin{array}{l}115,92 \mathrm{R} \\
86 ; 939 \mathrm{~T} \\
86,939 \mathrm{U}\end{array}$ & $\begin{array}{r}161,30 R \\
101 ; 061 \mathrm{~T} \\
101,061 \mathrm{U}\end{array}$ & $\begin{array}{l}119.26 \mathrm{R} \\
33,105 \mathrm{~T} \\
33,105 \mathrm{U}\end{array}$ & $\begin{array}{l}91,96 \mathrm{R} \\
76,487 \mathrm{~T} \\
76,487 \mathrm{U}\end{array}$ & $\begin{array}{r}95.03 \mathrm{R} \\
72,823 \mathrm{~T} \\
72,823 \mathrm{U}\end{array}$ & $\left.\left|\begin{array}{r}248.11 R \\
120,861 \mathrm{~T} \\
96,861 \mathrm{U}\end{array}\right|\right]$ & $\begin{array}{r}200,86 \mathrm{R} \\
130,880 \mathrm{~T} \\
110,8800\end{array}$ & $\begin{array}{r}138.91 R \\
112,409 \mathrm{~T} \\
112,4090 \\
\end{array}$ & $\begin{array}{l}114.11 \mathrm{~F} \\
53 ; 8297 \\
53 ; 8294\end{array}$ & $\begin{array}{r}92,26 \mathrm{P} \\
63,4271 \\
63,4270 \\
\end{array}$ & $\begin{array}{l}30.291 \\
3,352 \pi \\
3,3524 \\
\end{array}$ & $\begin{array}{r}85,39 \mathrm{R} \\
53,197 \mathrm{~T} \\
53,1970 \\
\end{array}$ & & \\
\hline
\end{tabular}


Experimental Users' Operations Summary

Counter Experiments

January through June 1966

\begin{tabular}{|c|c|c|c|c|c|c|c|c|c|c|c|c|}
\hline \multirow{2}{*}{$\begin{array}{l}\text { Hours test } \\
\text { Hours run } \\
\text { Hours chgd } \\
\end{array}$} & \multicolumn{4}{|c|}{ and } & \multicolumn{8}{|c|}{ Experiment Number } \\
\hline & $\begin{array}{c}153 \\
\text { coms. ilas }\end{array}$ & $\begin{array}{c}189 \\
\text { come } 3 / 16\end{array}$ & 210 & 245 & 269 & $\begin{array}{c}272 \\
\text { com? } 4 / 20\end{array}$ & $\begin{array}{c}275 \\
\text { comp } 4 / 27\end{array}$ & 280 & 281 & Biology & $\begin{array}{c}\text { Radio } \\
\text { Chemistry }\end{array}$ & \\
\hline $1 / 1-1 / 25$ & $\begin{array}{r}6.15 \mathrm{~T} \\
231.70 \mathrm{R} \\
189.3 \mathrm{c}\end{array}$ & $\begin{array}{r}31.92 \mathrm{~T} \\
300.32 \mathrm{R} \\
109.0 \mathrm{C}\end{array}$ & $\begin{array}{c}301.31 \mathrm{~T} \\
--- \\
---\end{array}$ & $=$ & 40 & $\begin{array}{l}90.73 T \\
--- \\
---\end{array}$ & $\because \because$ & $\begin{array}{c}100.54 \mathrm{~T} \\
--- \\
--\end{array}$ & & & 3.01 & \\
\hline $1 / 26-2 / 25$ & & $\begin{array}{l}-\overline{-}- \\
408.41 R \\
262.0 \mathrm{C}\end{array}$ & $\begin{array}{r}223.98 \mathrm{~T} \\
98.93 \mathrm{R} \\
44.5 \mathrm{C}\end{array}$ & & & $\begin{array}{r}7.25 \mathrm{~T} \\
405.55 \mathrm{R} \\
263.7 \mathrm{C}\end{array}$ & $\begin{array}{l}16.60 \mathrm{~T} \\
--- \\
---\end{array}$ & $\begin{array}{c}254.05 \mathrm{~T} \\
--- \\
---\end{array}$ & $=$ & 1.60 & 6.54 & \\
\hline $2 / 26-3 / 25$ & & $\begin{array}{r}223.71 \mathrm{~T} \\
.99 .75 \mathrm{R} \\
83.0 \mathrm{C}\end{array}$ & $\begin{array}{l}129.61 \mathrm{~T} \\
230.43 \mathrm{R} \\
149.2=\mathrm{C}\end{array}$ & & & $\begin{array}{l}-\frac{-}{-1} \\
98.47 R \\
93.1 \mathrm{C}\end{array}$ & $\begin{array}{r}288.17 \mathrm{~T} \\
38.4 \mathrm{IR} \\
37.4 \mathrm{C}\end{array}$ & $\therefore$ & & & & \\
\hline $3 / 26-4 / 25$ & & & $\begin{array}{r}38.87 \mathrm{~T} \\
204.72 \mathrm{R} \\
144.9 \mathrm{C}\end{array}$ & & & $\begin{array}{l}-\overline{-}-\overline{\mathrm{R}} \\
98.76 \mathrm{C} \\
93.1 \mathrm{C}\end{array}$ & $\begin{array}{r}97.95 \mathrm{~T} \\
338.2 \mathrm{IR} \\
321.5 \mathrm{C}\end{array}$ & & & & r & \\
\hline $4 / 26-5 / 25$ & & & $\begin{array}{r}26.62 \mathrm{~T} \\
298.60 \mathrm{R} \\
253.8 \mathrm{C} \\
\end{array}$ & $\begin{array}{r}96.84 \mathrm{~T} \\
208.47 \mathrm{R} \\
179.9 \mathrm{C} \\
\end{array}$ & $\begin{array}{l}94.50 \mathrm{~T} \\
--- \\
--- \\
\end{array}$ & & $\begin{array}{l}--1- \\
29.48 \mathrm{R} \\
29.5 \mathrm{C} \\
\end{array}$ & & $\begin{array}{l}49.23 \mathrm{~T} \\
--- \\
---\end{array}$ & & 3.78 & \\
\hline $5 / 26-6 / 30$ & & & $\begin{array}{l}10.00 \mathrm{~T} \\
52.7 \mathrm{LR} \\
52.6 \mathrm{C}\end{array}$ & & $\begin{array}{c}222.44 \mathrm{~T} \\
=-- \\
-= \\
\end{array}$ & & . & & $\begin{array}{c}214.39 \mathrm{~T} \\
--- \\
--- \\
\end{array}$ & & 1.66 & \\
\hline $\begin{array}{l}\text { Totals } \\
\text { Jan thru } \\
\text { Jun. } 66\end{array}$ & $\begin{array}{r}6.15 \mathrm{~T} \\
231.70 \mathrm{R} \\
189.3 \mathrm{C} \\
\end{array}$ & $\begin{array}{l}255.63 \mathrm{~T} \\
808.48 \mathrm{R} \\
454.0 \mathrm{C}\end{array}$ & $\begin{array}{l}730.39 \mathrm{~T} \\
885.42 \mathrm{R} \\
645.0 \mathrm{C} \\
\end{array}$ & $\begin{array}{r}96.84 \mathrm{~T} \\
208.47 \mathrm{R} \\
179.9 \mathrm{C}\end{array}$ & $\begin{array}{c}216.94 \mathrm{~T} \\
--- \\
-- \\
\end{array}$ & $\begin{array}{r}97.98 \mathrm{~T} \\
602.78 \mathrm{R} \\
445.7 \mathrm{C} \\
\end{array}$ & $\begin{array}{l}402.72 \mathrm{~T} \\
406.10 \mathrm{R} \\
388.4 \mathrm{C}\end{array}$ & $\begin{array}{c}354.59 \mathrm{~T} \\
-- \\
-- \\
\end{array}$ & $\begin{array}{c}263.62 \mathrm{~T} \\
--- \\
-- \\
\end{array}$ & 1.60 & 13.33 & \\
\hline $\begin{array}{l}\text { Previous } \\
\text { Totals } \\
\vdots\end{array}$ & $\begin{array}{l}221.63 \mathrm{~T} \\
886.38 \mathrm{R} \\
608.7 \mathrm{C} \\
\end{array}$ & $\begin{array}{c}114.38 \mathrm{~T} \\
--- \\
--- \\
\end{array}$ & $\begin{array}{r}778.13 \mathrm{~T} \\
-\div- \\
\end{array}$ & $\begin{array}{l}225.27 \mathrm{~T} \\
184.11 \mathrm{R} \\
183.00\end{array}$ & & & & & & & & \\
\hline $\begin{array}{l}\text { Totals } \\
\text { to date }\end{array}$ & $\begin{array}{r}227.78 \mathrm{~T} \\
1118.08 \mathrm{R} \\
798.0 \mathrm{C}\end{array}$ & $\begin{array}{l}370.01 T \\
808.48 \mathrm{R} \\
454.0 \mathrm{C} \\
\end{array}$ & $\begin{array}{r}1508.52 \mathrm{~T} \\
885.42 \mathrm{R} \\
645.0 \mathrm{C} \\
\end{array}$ & $\begin{array}{l}322.11 \mathrm{~T} \\
392.58 \mathrm{R} \\
362.9 \mathrm{C} \\
\end{array}$ & $\begin{array}{c}316.94 \mathrm{~T} \\
--- \\
-- \\
\end{array}$ & $\begin{array}{r}97.98 \mathrm{~T} \\
602.78 \mathrm{R} \\
445.7 \mathrm{C} \\
\end{array}$ & $\begin{array}{l}402.72 \mathrm{~T} \\
406.10 \mathrm{R} \\
388.4 \mathrm{C}\end{array}$ & $\begin{array}{c}354.59 \mathrm{~T} \\
--= \\
--= \\
\end{array}$ & $\begin{array}{r}263.62 \mathrm{~T} \\
--- \\
--- \\
\end{array}$ & 1.60 & 13.33 & \\
\hline
\end{tabular}


Experimental Users' Operations Summary

Counter Experiments

July through December 1966

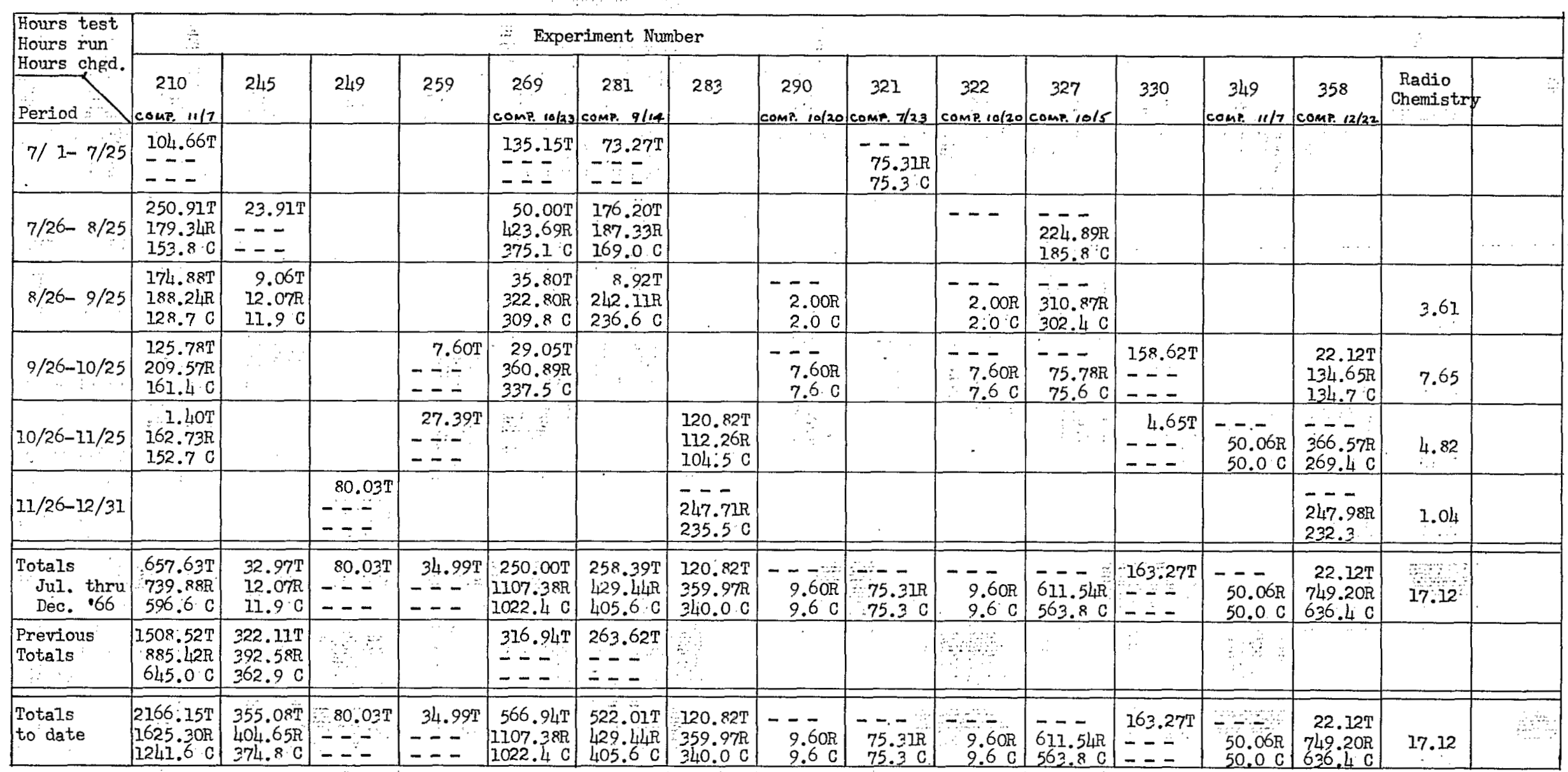


AGS OPERATIONS SUMMARY

1964, 1965, 1966, Yearly Comparisons

\section{AGS ON}

1. For Experimenters

2. For Accelerator Research MACHINE DOWNTTME

1. Machine Set-up, Shutdown

2. Requested Down

3. Experimental Set-up

4. Machine Failures

5. EAO Equipment Failures

6. Scheduled Mairitenance

SCHEDULED OPERATIONS

\section{HOLIDAYS}

SHUTLONN

\section{AGS PERFORMANCE}
a. Magnet pulses
b. Accelerated pulses
c. Missed pulses
d. Accelerated protons ( $p$ )
e. Aver. $p / p u l s e(d / b)$
f. Aver. $p /$ pulse $(d / b+c)$

4

\section{4}

$\frac{\text { Hours }}{4.762 .02 \quad 0 \%}$

$4.196 .26 \quad 61.9$

565.76

$2,016.98$

332.75

70.70

0.00

$1,119.94$

88.25

$\frac{405.34}{6.779 .00} \quad \frac{6.0}{100.0}$

156.00

$1,849.00$

$6,340,509$

N.A.

$2.3 \times 10^{18}$

$3.6 \times 10^{11}$

N.A.

\begin{tabular}{rr}
\multicolumn{2}{c}{1965} \\
\hline Hours & $\% / 0$ \\
\hline 4.623 .78 & 71.5 \\
$3,836.00$ & 59.3 \\
787.77 & 12.2 \\
1.840 .35 & 28.5 \\
233.13 & 3.6 \\
61.81 & 1.0 \\
27.12 & 0.4 \\
783.45 & 12.1 \\
120.19 & 1.9 \\
614.65 & 9.5 \\
\hline 6.464 .13 & 100.0 \\
240.00 &
\end{tabular}

$2,055.87$

$7,286,034$

$5,638,403$

483,912

$4.0 \times 10^{18}$

$7.1 \times 10^{11}$

$6.5 \times 10^{11}$
1966

\begin{tabular}{rr}
\hline Hours & \multicolumn{1}{c}{0} \\
\hline 5.671 .89 & 75.8 \\
4.991 .27 & 66.7 \\
680.62 & 9.1 \\
1.812 .11 & 24.2
\end{tabular}

$273.04 \quad 3.7$

58.17

0.8

31.75

0.4

833.22

11.1

76.02

1.0

539.91

$7,484.00$

$\frac{7.2}{100.0}$

212.00

$1,064.00$

$8,916,874$

$7,481,254$

538,542

$8.7 \times 10^{18}$

$11.6 \times 10^{11}$

$10.9 \times 10^{11}$ 
AGS OPERATIONS SUMMARY

1964, 1965, 1966, 1/2 Year Comparison

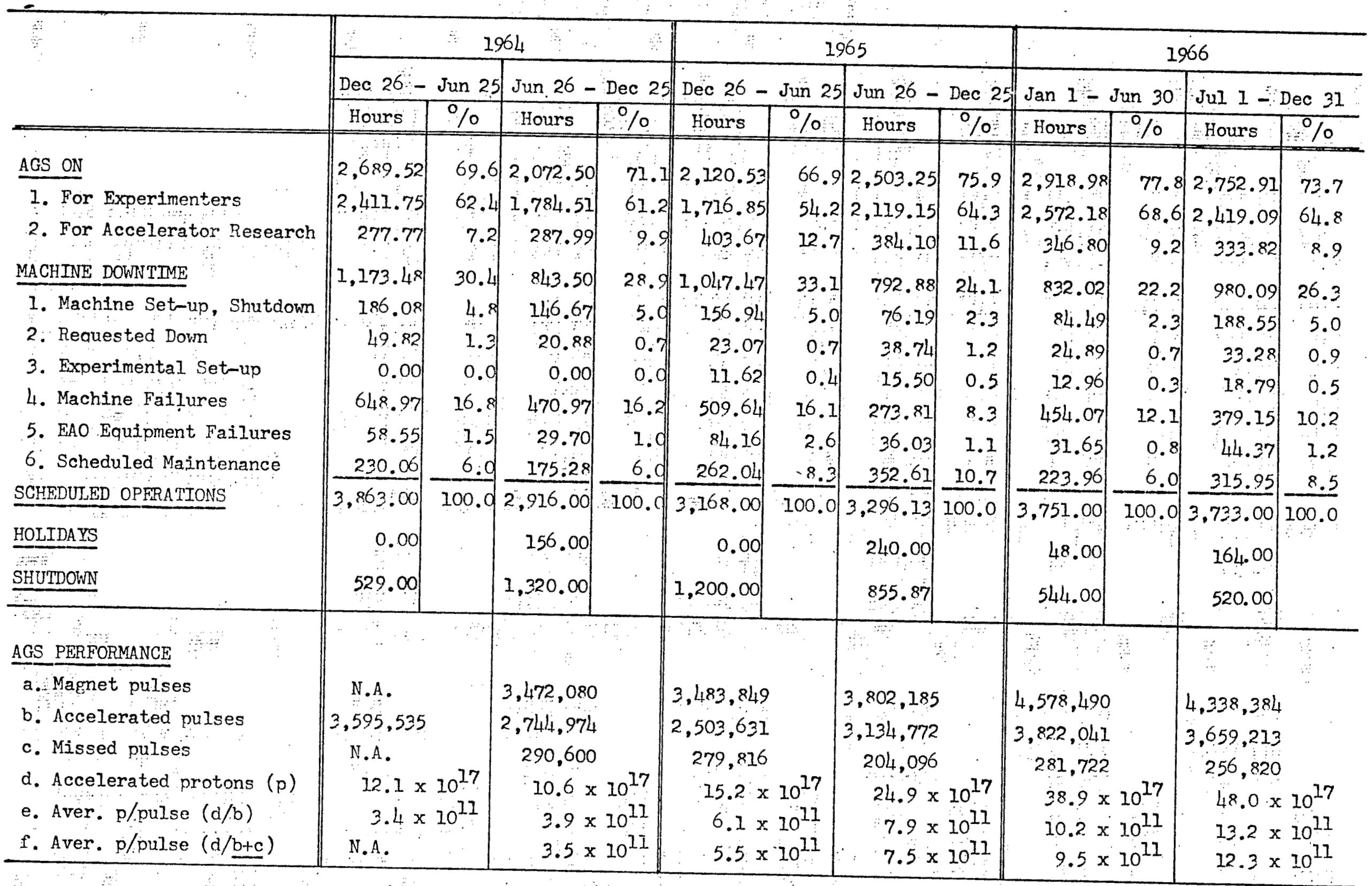

